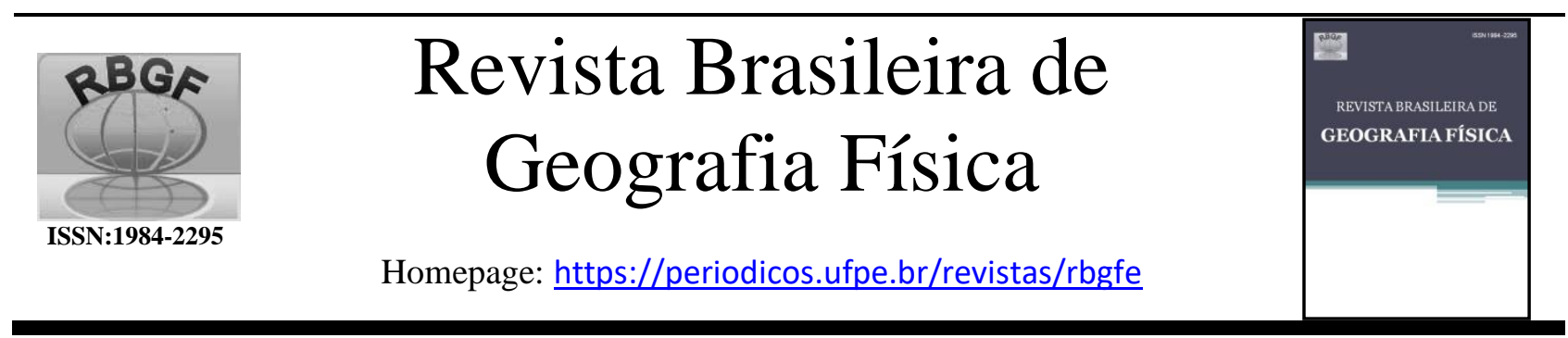

\title{
Análise de tendência das componentes do saldo de radiação e número de horas de frio em Cruz Alta-RS
}

\author{
Rodrigo Lins da Rocha Júnior ${ }^{1}$, Fabrício Daniel dos Santos Silva ${ }^{2}$, Rafaela Lisboa Costa ${ }^{3}$, \\ Heliofábio Barros Gomes ${ }^{4}$, William Max de Oliveira Romão ${ }^{5}$
}

\begin{abstract}
${ }^{1}$ Mestrando em Meteorologia, Instituto de Ciências Atmosféricas, Universidade Federal de Alagoas, Av. Lourival Melo Mota, s/n, Tabuleiro do Martins, CEP:57072-900, Maceió-AL. (82) 3214-1367. rrodrigo.junior@ hotmail.com. ${ }^{2}$ Professor Adjunto, Instituto de Ciências Atmosféricas, Universidade Federal de Alagoas, Av. Lourival Melo Mota, s/n, Tabuleiro do Martins, CEP:57072-900, Maceió-AL. (82) 3214-1367. fabricio.santos@icat.ufal.br. (autor correspondente). ${ }^{3}$ Dra. em Geociências Aplicadas, Bolsista PNPD/CAPES, Instituto de Ciências Atmosféricas, Universidade Federal de Alagoas, Av. Lourival Melo Mota, s/n, Tabuleiro do Martins, CEP:57072-900, Maceió-AL. (82) 3214-1367. rafaelalisboac@ gmail.com. ${ }^{4}$ Professor Associado, Instituto de Ciências Atmosféricas, Universidade Federal de Alagoas, Av. Lourival Melo Mota, s/n, Tabuleiro do Martins, CEP:57072-900, MaceióAL. (82) 3214-1367. heliofabio@ icat.ufal.br. ${ }^{5}$ Graduando em Meteorologia, Instituto de Ciências Atmosféricas, Universidade Federal de Alagoas, Av. Lourival Melo Mota, s/n, Tabuleiro do Martins, CEP:57072-900, Maceió-AL. (82) 3214-1367.william.romao@icat.ufal.br.
\end{abstract}

Artigo recebido em 05/08/2019 e aceito em 01/03/2020

\section{RES U M O}

Esta pesquisa avaliou as tendências do balanço de ondas curtas (BOC), balanço de ondas longas (BOL) e do saldo de radiação (SR), para relacionar com a tendência observada do número de horas de frio (NHF) para a cidade de Cruz AltaRS. Foram utilizados dados horários da estação meteorológica automática do Instituto Nacional de Meteorologia no período de 2010 a 2018. As análises foram relativas aos períodos das estações do ano, e para obtenção das tendências foi utilizado o teste não paramétrico de Mann-Kendall. O BOC e o BOL apresentaram tendências positivas no inverno, e negativas no verão e outono. O SR apresentou tendências negativas no outono, primavera e verão, e positiva no inverno. Não foram observada horas de frio no verão. No outono houve a tendência de aumento estatisticamente significante do NHF, enquanto no inverno e primavera as tendências foram de redução do NHF, com significância estatística no inverno. A tendência de redução de $-1,39$ horas de frio no inverno e de $-0,11$ horas de frio na primavera podem estar associadas a redução significativa da passagem de frentes frias e da atuação de massas de ar frio na região sul do Brasil nos últimos anos da análise. Tais resultados precisam de atenção, pois é principalmente no inverno que deve ocorrer o acúmulo natural de NHF para diversas culturas. Em geral, anos com NHF reduzido estão associados aos piores índices de produtividade das culturas que necessitam acumular horas de frio.

Palavras-chave: radiação, temperatura, agricultura.

\section{Trend analysis of the radiation balance components and number of cold hours in Cruz Alta-RS}

\begin{abstract}
A B S T R A C T
This research evaluated the shortwave balance (BOC), longwave balance (BOL) and net radiation (SR) trends to correlate with the observed trend of chill hours (NHF) for the city of Cruz Alta-RS. Hourly data from the INMET automatic weather station were used from 2010 to 2018. The analyzes were relative to the seasons, and to obtain the trends, the nonparametric Mann-Kendall test was used. BOC and BOL showed positive trends in winter and negative trends in summer and fall. SR showed negative trends in fall, spring and summer, and positive trends in winter. No cold hours were observed in summer. In the fall there was a trend of statistically significant increase in NHF, while in winter and spring the trends were in NHF reduction, with statistical significance in winter. The tendency to reduce -1.39 chill hours in winter and -0.11 chill hours in spring may be associated with a significant reduction in cold front passage and cold air masses in southern Brazil in the last years of analysis. These results need attention, as it is mainly in winter that natural accumulation of NHF should occur for several crops. In general, years with reduced NHF are associated with worse crop yields that need to accumulate chili hours.
\end{abstract}

Keywords: radiation, temperature, agriculture. 


\section{Introdução}

O estudo dos componentes do saldo de radiação (SR) de uma superfície cultivada constitui-se em uma variável fundamental na modelagem dos fluxos de propriedades físicas, como a massa e a energia na camada limite planetária. Sobre a magnitude do saldo de radiação interferem diversos fatores relacionados com as ondas curtas e ondas longas. Destacam-se latitude, altitude, época do ano, cobertura de nuvens, composição espectral da radiação incidente, propriedades espectrais da cultura, grau de cobertura do solo ela cultura, disponibilidade hídrica no solo e temperatura da superfície e da atmosfera (Blad e Baker, 1972; Angelini et al., 2017).

A capacidade de absorção do SR derivado do balanço entre ondas curtas e longas pelo meio depende fortemente do tipo de cobertura vegetal (Fausto et al., 2014; Biudes et al., 2015), e da disponibilidade de água no sistema (Cunha et al., 2012; Matins et al., 2015). No entanto, há diversas culturas que necessitam, além do acúmulo de energia, acumular horas de frio durante o inverno para que entrem em estágio de dormência, e depois de completada a exigência em frio, ocorrer a brotação e a floração (Leite et al., 2004; Botelho, 2006).

O termo "horas de frio" refere-se ao número acumulado de horas com temperaturas inferiores a um valor crítico, que pode ser de $7^{\circ} \mathrm{C}$, válida para a maioria das plantas criófilas mais exigentes ao frio, como por exemplo uva, ameixa, maçã, pêssego, figo, caqui e kiwi, entre outras. Para plantas um pouco menos exigentes ao frio, esta temperatura crítica gira em torno dos $13^{\circ} \mathrm{C}$ (Martin, 2012).

É importante conhecer a quantidade de horas de frio que determinada área recebe com os requisitos de horas de frio das variedades de frutas que pretende-se cultivar. Caso contrário, as árvores quebrarão a dormência muito cedo (no caso da maçã com pouco frio) ou tarde demais para uma polinização adequada e estarão mais sujeitas a problemas de doenças (Bradley e Maurer, 2002).

Durante a dormência, uma árvore não retoma o crescimento até atingir as horas de frio necessárias. Essa estratégia adaptativa confere a capacidade de resistir a estímulos externos do ambiente para crescer em dias quentes de inverno ou durante as geadas de inverno, e para aguentar seu tempo antropomórfico até que condições favoráveis de crescimento ocorram na primavera (Bradley e Maurer, 2002; Martin, 2012).
Na região Sul do Brasil, tal situação é determinante para que culturas como a macieira, pereira e pessegueiro, por exemplo, atinjam condições plenas de produtividade (Bianchi et al., 2000; Hauage e Cummins, 2000; Citadin et al., 2002; Nienow e Floss, 2002; Nachtigall, 2004; Pedro Júnior et al., 2007).

No caso específico do cômputo do número de horas de frio (NHF), um fator limitante até o início dos anos 2000 era a pequena disponibilidade de estações meteorológicas automáticas (EMA) que permitissem a coleta horária de dados. Entretanto, a partir de 2000 uma série de EMA's passou a ser instalada pelo Instituto Nacional de Meteorologia (INMET), concomitante á estações meteorológicas convencionais, assim como também em localidades que não possuíam nenhuma estação.

Conhecendo-se a importância dos parâmetros constituintes do SR e do acúmulo de horas de frio para a produtividade de culturas que dependem diretamente destes fatores, o objetivo desta pesquisa foi correlacionar a evolução temporal das tendências da radiação de ondas curtas e longas, assim como do NHF na cidade de Cruz Alta, no estado do Rio Grande do Sul, no período recente de 2010 a 2018.

\section{Material e métodos}

Dados e Área de Estudo

Foram utilizados os dados horários da EMA do INMET localizada no município de Cruz Alta (RS), para o período ininterrupto de 2010 a 2018. A Figura 1 mostra a localização do município no Estado, na mesorregião do noroeste rio-grandense. A EMA está situada a $-28.60^{\circ}$ de latitude, $-53.67^{\circ}$ de longitude, a uma altitude de 427 metros.

O clima em Cruz Alta, segundo a classificação de Koppen, é o Cfa ou clima subtropical úmido, que tem entre suas características temperaturas amenas e pode apresentar temperaturas médias entre $-3^{\circ} \mathrm{C}$ a acima de $0^{\circ} \mathrm{C}$ nos meses mais frios.

Para calcular o BOC e o BOL foram utilizados os dados de temperatura, umidade relativa e radiação solar, sendo o SR a resultante do somatório dos dois balanções radiativos, como segue: 


$$
B O C=\operatorname{Rg}(1-\alpha)
$$

em que $\operatorname{Rg}$ é a radiação solar global e $\alpha$ é o albedo da superfície, onde foi utilizado o valor médio para gramado $=0,23$.

Como a EMA do INMET disponibiliza Rg em $\mathrm{KJ} / \mathrm{m}^{2} /$ dia, para sua conversão em $\mathrm{MJ} / \mathrm{m}^{2} / \mathrm{dia}$ basta a divisão por 1000 . Valores similares são obtidos quando comparados a equação média obtida por Fontana et al (1996) a partir de 21 estações meteorológicas do Rio Grande do Sul. O BOL é obtido da equação que relaciona a perda de radiação por ondas longas pela superfície e reemitida pela atmosfera à superfície, como segue:

$B O L=O L \uparrow+O L \downarrow$

$B O L=-\varepsilon_{a} \sigma T_{a}^{4}+\varepsilon_{S} \sigma T_{S}^{4}$

$B O L=-\sigma T^{4}\left(0,325-0,139 e^{0,5}\right)\left(0,1+0,9 \frac{n}{N}\right)$

em que $\varepsilon$ é a emissividade; $\sigma$ é a constante de Stefan-Boltzman $\left(4,9 \mathrm{x} 10-9 \mathrm{MJ} / \mathrm{m}^{2} / \mathrm{dia}^{\circ} \mathrm{K}^{-4}\right)$; T é a temperatura $(\mathrm{K})$; s e a são referentes ao solo e ar, respectivamente. Levando-se em consideração que a temperatura do ar é aproximadamente igual a temperatura do solo em um período de 24 , e que a emissividade do solo é aproximadamente 1, tem-se a equação (2) para o cálculo do BOL, onde e é a pressão parcial do vapor d'água, $n$ é a insolação que foi obtida da estação meteorológica convencional do INMET em Cruz Alta e N é o fotoperíodo. Neste trabalho, para uma melhor visualização dos resultados, mostra-se o valor absoluto para BOL nos gráficos das Figuras 2 a 5, assim, o SR mostrado nas figuras é a resultante de BOC-BOL.

Para computar o NHF foram utilizados os dados horários de temperatura da EMA. O BOC e o BOL foram calculados e tiveram as respectivas tendências analisadas para as quatro estações do ano, enquanto o NHF não foi analisado apenas para o verão. No caso do NHF, segundo Pedro Júnior et al. (1979) a temperatura média do ar de $7^{\circ} \mathrm{C}$ é o limite térmico superior para o cômputo do NHF por ser o valor máximo de temperatura necessárias na fase de dormência das plantas criófilas. Desta forma, o valor de $7^{\circ} \mathrm{C}$ foi utilizado como limiar para o cálculo do NHF. 


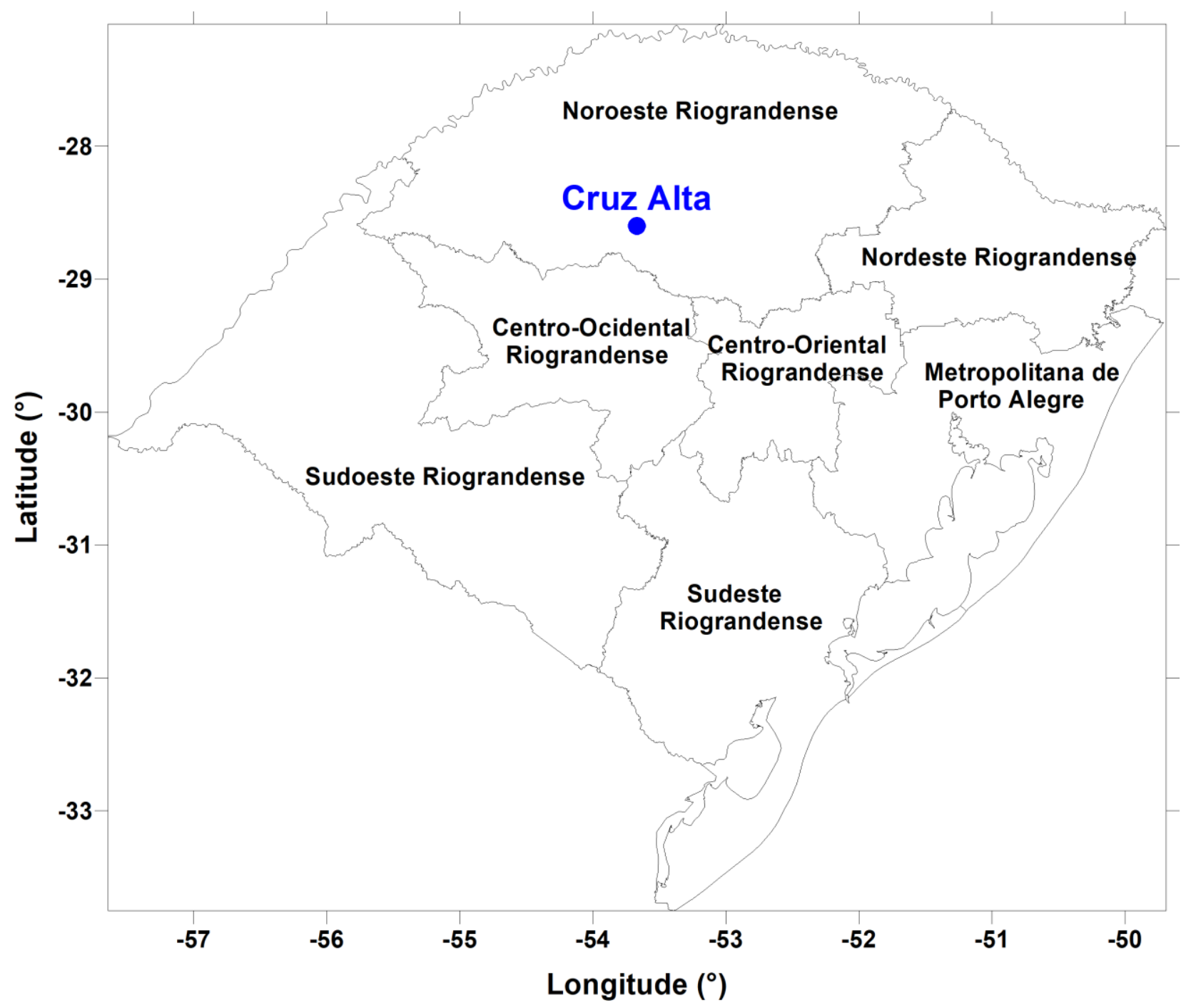

Figura 1. Localização do município de Cruz Alta, na mesorregião do noroeste rio-grandense.

Análise de Tendência

Para a análise das tendências foi usado o teste não-paramétrico de Mann-Kendall (MK), aplicado aos dados diários do BOC, BOL e NHF. $\mathrm{O}$ teste $\mathrm{MK}$ mostra se as tendências estatísticas são aleatórias ou não, sem a necessidade de conhecimento prévio da distribuição dos dados (Sen., 1968; Richard., 1987; Salmi, et al., 2002; Santos et al., 2016), sendo considerado o mais adequado para análise de tendências em séries temporais climáticas (Onoz and Bayazit., 2003; Machiwal and Jha., 2008; Some'e et al., 2013). O teste MK geralmente é aplicado considerando a estatísitca S, como em Modarres e da Silva (2007) (3).

$$
S=\sum_{i=2}^{n} \sum_{j=1}^{i-1} \operatorname{sign}\left(x_{i}-x_{j}\right)
$$

nde $\mathrm{X}_{\mathrm{i}}$ é o valor sequencial dos dados, $\mathrm{n}$ é o tamanho da série temporal e $\operatorname{sign}\left(X_{i}-X_{j}\right)=-1$ se $\left(\mathrm{X}_{\mathrm{i}}-\mathrm{X}_{\mathrm{j}}\right)<0 ; \operatorname{sign}\left(\mathrm{X}_{\mathrm{i}}-\mathrm{X}_{\mathrm{j}}\right)=0$ se $\left(\mathrm{X}_{\mathrm{i}}-\mathrm{X}_{\mathrm{j}}\right)=0$ e
$\operatorname{sign}\left(X_{i}-X_{j}\right)=1$ se $\left(X_{i}-X_{j}\right)>0$. A hipótese nula $\mathrm{H}_{0}$ é que uma amostra de dados $\left\{\mathrm{X}_{\mathrm{t}}: \mathrm{t}=1,2, \ldots, \mathrm{n}\right\} \mathrm{i}$ é independente e identicamente distribuído. A hipótese alternativa $\mathrm{H}_{1}$ é que existe uma tendência monotônica em $\left\{\mathrm{X}_{\mathrm{t}}\right\}$. Cada par de valores observados $\left(\mathrm{X}_{\mathrm{i}}, \mathrm{X}_{\mathrm{j}}\right)$ onde $\mathrm{i}>\mathrm{j}$ é inspecionada para encontrar $X_{i}>X_{j}$ (primeiro tipo) ou $X_{i}<X_{j}$ (segundo tipo). A esperança $E[S]$ e variância Var[S] da estatística S são obtidas por (4) e (5):

$$
\begin{gathered}
E[S]=0 \text { (4) } \\
\operatorname{Var}[S]=\frac{n(n-1)(2 n+5)-\sum_{p=1}^{q} t_{p}\left(t_{p}-1\right)\left(2 t_{p}+5\right)}{18}
\end{gathered}
$$

em que $n$ é o número de observações e, considerando o caso em que a série pode ter grupos 
com observações iguais, $\mathrm{P}$ é o número de grupos com observações iguais e tP é o número de observações iguais no grupo P. Um distribuição normal da variável $\mathrm{Z}$ é construída como mostra as equações em (6):

$$
Z=\left\{\begin{array}{l}
\frac{S-1}{\sqrt{\operatorname{Var}(S)}}, S>0 \\
0, S=0 \\
\frac{S+1}{\sqrt{\operatorname{Var}(S)}}, S<0
\end{array}\right.
$$

Em um teste duplo para a tendência, a hipótese nula de não tendência é rejeitada se $|Z|>$ $Z \alpha / 2$, onde $\alpha$ é o nível de significância. Dada a grande variabilidade dos dados, consideramos tendências estatisticamente significantes quando o p-valor for igual ou menor a $10 \%$. Mais detalhes sobre o método MK, recomenda-se a leitura de Wilks (2011).

\section{Resultados e discussão}

A análise das componentes e do SR iniciase para o outono, tendo sido estudados os nove outonos completos em sequência de 2010 a 2018. Neste período, tanto o BOC (Figura 2a) quanto o BOL (Figura 2b), mostram tendências negativas e estatisticamente significantes de $-0,0034$ $\mathrm{MJ} / \mathrm{m}^{2} / \mathrm{dia} /$ ano e $-0,0013 \mathrm{MJ} / \mathrm{m}^{2} / \mathrm{dia} /$ ano. Este comportamento resultou em tendência de diminuição do SR da ordem de $-0,0021$ $\mathrm{MJ} / \mathrm{m}^{2} /$ dia/ano (Figura 2c).

A Figura 3 mostra as tendências para o inverno. Mesmo sendo a estação sequencial ao outono, o observado nestes últimos 9 invernos entre 2010 a 2018 foram tendências positivas para o BOC (Figura 3a) e BOL (Figura 3b), de $0,00051 \mathrm{MJ} / \mathrm{m}^{2} / \mathrm{dia} /$ ano e $0,00047 \mathrm{MJ} / \mathrm{m}^{2} / \mathrm{dia} /$ ano. Apenas a tendência do BOL apresentou significância estatística. No entanto, o SR teve comportamento quase linear, com tendência sem significância estatística, positiva, da ordem de $0,00008 \mathrm{MJ} / \mathrm{m}^{2} / \mathrm{dia} /$ ano (Figura $3 \mathrm{c}$ ).

$\mathrm{Na}$ primavera, foram observadas tendências negativas de BOC (Figura 4a) e BOL (Figura $4 \mathrm{~b}$ ), da ordem de $0,001 \mathrm{MJ} / \mathrm{m}^{2} / \mathrm{dia}$ e positivas do BOL, de $0,0004 \mathrm{MJ} / \mathrm{m}^{2} / \mathrm{dia} / \mathrm{ano}$, sem significância estatística para o BOL. Com BOC < BOL, o SR resultante mostrou-se com tendência negativa e estatisticamente significante, da ordem de $0,0012 \mathrm{MJ} / \mathrm{m}^{2} / \mathrm{dia} /$ ano (Figura $4 \mathrm{c}$ ).

No verão, ambas as tendências de BOC (Figura 5a) e BOL (Figura 5b) foram negativas e estatisticamente significantes. O BOC apresentou tendência da ordem de $0,0011 \mathrm{MJ} / \mathrm{m}^{2} / \mathrm{dia} / \mathrm{ano}$ e o BOL de $0,002 \mathrm{MJ} / \mathrm{m}^{2} /$ dia/ano. A tendência mais Da Rocha Júnior, R. L.; Silva, F. D. S.; Costa, R. L.; Gomes, H. B. G.; Romão, W. M.. pronunciada do BOL resultou em SR com tendência positiva e estatisticamente significativa da ordem de $0,0009 \mathrm{MJ} / \mathrm{m}^{2} / \mathrm{dia} /$ ano (Figura $5 \mathrm{c}$ ).

Analisando-se as tendências do NHF, a Figura 6 mostra o comportamento dos últimos 9 anos para a primavera (Figura 6a), outono (Figura $6 \mathrm{~b})$ e inverno (Figura 6c). Os resultados para os verões não são mostrados por não ter sido verificado NHF significativas para análise de tendências.

$\mathrm{Na}$ primavera (Figura 6a) a tendência foi negativa, de -0,11NHF, mas sem significância estatística. Este resultado evidencia a influência não tanto do SR com tendência negativa, mas principalmente das tendências negativas do BOC, superiores em magnitude as tendências positivas do $\mathrm{BOL}$, diminuindo assim as chances de decréscimos mais acentuados das temperaturas durante as horas do dia sob o brilho solar e a maior perda de energia da superfície para o espaço durante a noite.

No outono (Figura 6b), a tendência positiva significativa do NHF foi de 0,68 , ou aproximadamente 6 horas de frio no período. A tendência negativa do BOC reflete a menor retenção de energia durante o dia favorecendo a queda das temperaturas.

No inverno (Figura 3), as componentes do SR, BOC e BOL, apresentaram tendências positivas, evidenciando a maior entrada de energia por radiação direta durante os dias, que mesmo balanceada pela tendência positiva do BOL, associada a perda radiativa noturna para o espaço em maior magnitude do que a que retorna devido a camada de nuvens e gases, resultou em SR positivo. Associado a isto observou-se no período a tendência de diminuição do NHF em até 1,39 horas ao longo dos invernos estudados. O inverno é a principal estação do ano para o acumulo natural de NHF para diversas culturas. A redução do NHF é fator preocupante, haja visto que as projeções futuras de mudanças climáticas são unanimes em apontar na direção do aumento das temperaturas, principalmente as temperaturas mínimas, podendo acarretar em invernos cada vez mais amenos com menor acumulação de NHF (Marengo, 2006).

Apesar destes resultados serem relativos a um período curto de dados, apenas 9 anos, ou nove estações do ano bem definidas analisadas, buscouse algumas explicações físicas para associar melhor as causas e efeitos avaliados. Foram analisados os números de frentes frias $(\mathrm{FF})$ e de massas de ar frio (MAF) que atingiram a região sul do Brasil de 2010 a 2018, e consequentemente a cidade de Cruz Alta. Estes dados puderam ser coletados nos boletins climanálise do CPTEC/INPE, de 2010 a 2014 (http://climanalise.cptec.inpe.br/ rclimanl/boletim ), último ano disponível, complementados pelos 
boletins agrometeorológicos mensais do INMET, $\begin{array}{llll}\text { de } & 2015 & \text { a } & 2018\end{array}$ (http://www.inmet.gov.br/portal/index.php?r=agro meteorologia/boletinsAgroclimatologicos).

A Figura 7 mostra a variabilidade do número de FF e MAF por estação do ano entre 2010 a 2018 na região sul do Brasil.

No outono, houve uma média de $14 \mathrm{FF}$ e 14 MAF. Entre as três estações do ano estudadas, no outono (Figura 7a), houve a mais leve tendência, quase linear e sem significância, de diminuição no número de FF e MAF, com destaque para os três últimos anos, 2016 a 2018. No entanto, no inverno e primavera a tendência observada de redução no número de FF e MAF foi significativa. No inverno (Figura $7 \mathrm{~b}$ ), houve tendência de $-7,8 \mathrm{FF} /$ período e de $-6,4 \mathrm{MAF} /$ período, um número expressivo que pode estar diretamente associado a menor queda das temperaturas e na consequente tendência de diminuição do NHF. Na primavera, que também apresentou tendência de diminuição no NHF, as tendências foram de aproximadamente 4,5FF/período e -8,8MAF (Figura 7c).

Estes resultados são fortes indícios de que o aumento ou diminuição da frequência de passagens de FF e incursões de MAF é o principal fator determinante nas tendências do SR e suas componentes, além do aumento e/ou diminuição do $\mathrm{NHF}$ a partir de $7^{\circ} \mathrm{C}$, essencial para $\mathrm{o}$ desenvolvimento de culturas exigentes a esta variável.
Estudos desta natureza são de extrema importância para tomadas de decisões no setor agrícola. Yoshikawa et al (2018) compararam dois modelos de estimativas do acúmulo de NHF em diferentes municípios da região sul do Brasil com a região de Aomori no Japão, para fins de viabilidade da produção de maçãs, com dados de 2000 a 2015. Os resultados indicaram, além do melhor modelo a ser aplicado, que no sul do Brasil há grande variação do NHF, com os locais mais favoráveis associados as maiores altitudes.

Milech et al (2018) comparam oito modelos de estimativa do NHF para 11 anos de dados de inverno em Pelotas-RS. Geirinhas et al (2017), mostraram que existe tendências positivas e significativas da frequência de ondas de calor desde a década de 80 nas diferentes regiões brasileiras.

Baldochi e Wong (2008) notaram a diminuição no NHF acumulados no inverno nas regiões produtoras de frutas e nozes da Califórnia, a partir de dados horários e modelos adequados ao cálculo do NHF a partir de temperaturas máximas e mínimas desde os anos 50, com 50 a 260 horas de frio a menos por década. Resultados similares de tendências de diminuição do NHF foram obtidos por Luedeling et al (2008) em quatro oásis de montanha em Omã, na Península Arábica. Além disso, estes estudos se preocuparam em demonstrar que essas tendências podem se agravar para diferentes cenários de mudanças climáticas futuras. 
a)

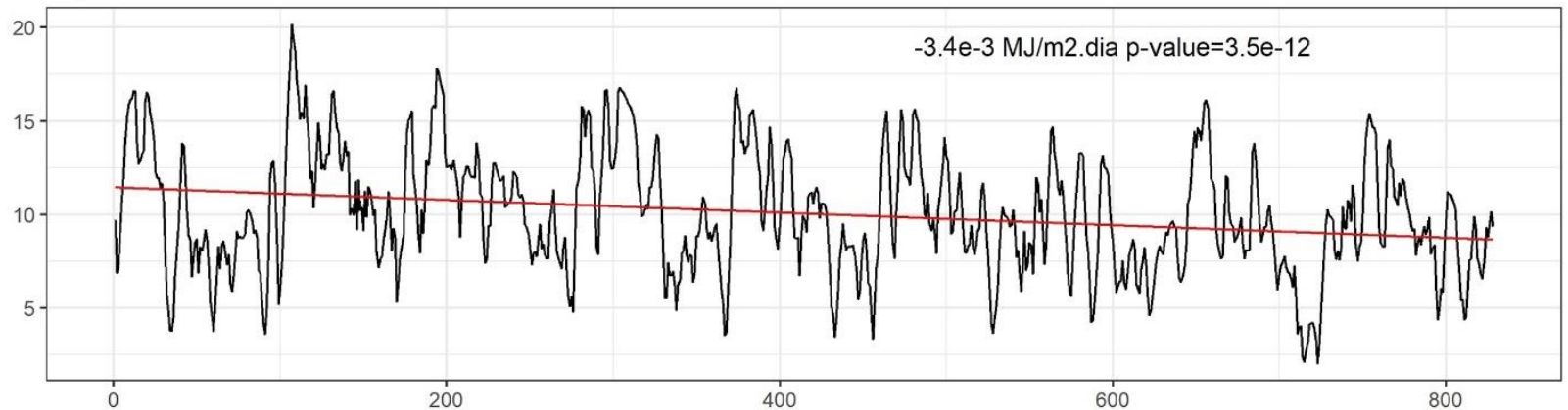

b)

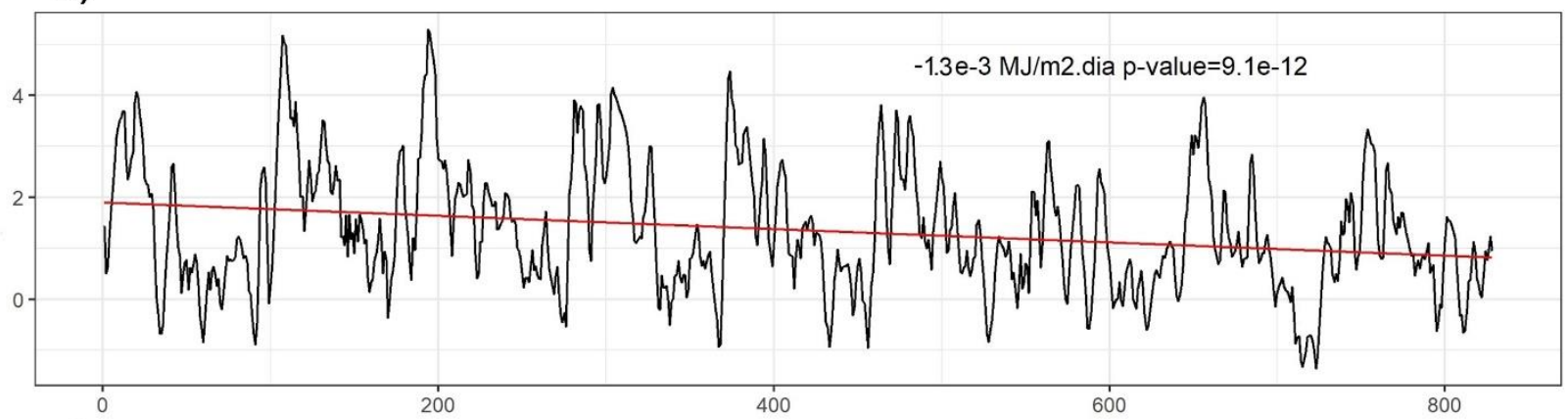

C)

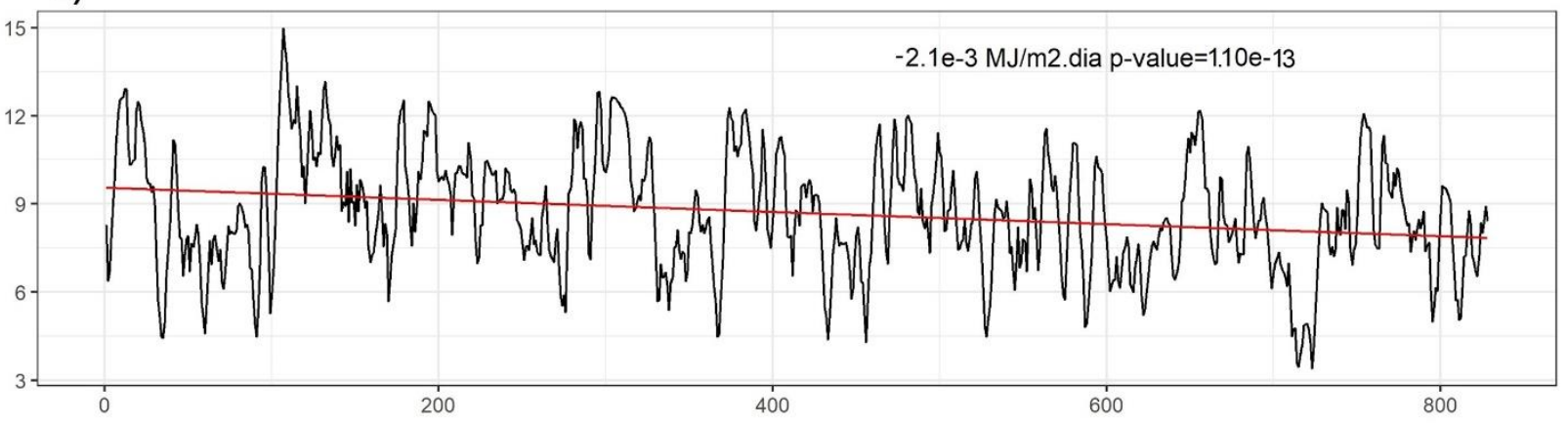

Figura 2. Tendência de (a) Balanço de Ondas Longas, (b) Balanço de Ondas Curtas e (c) Saldo de Radiação, no eixo y, em MJ/m2dia-1 durante os outonos em Cruz Alta no período 2010-2018 (em número de dias contínuos no eixo $\mathrm{x}$ ). 
a)

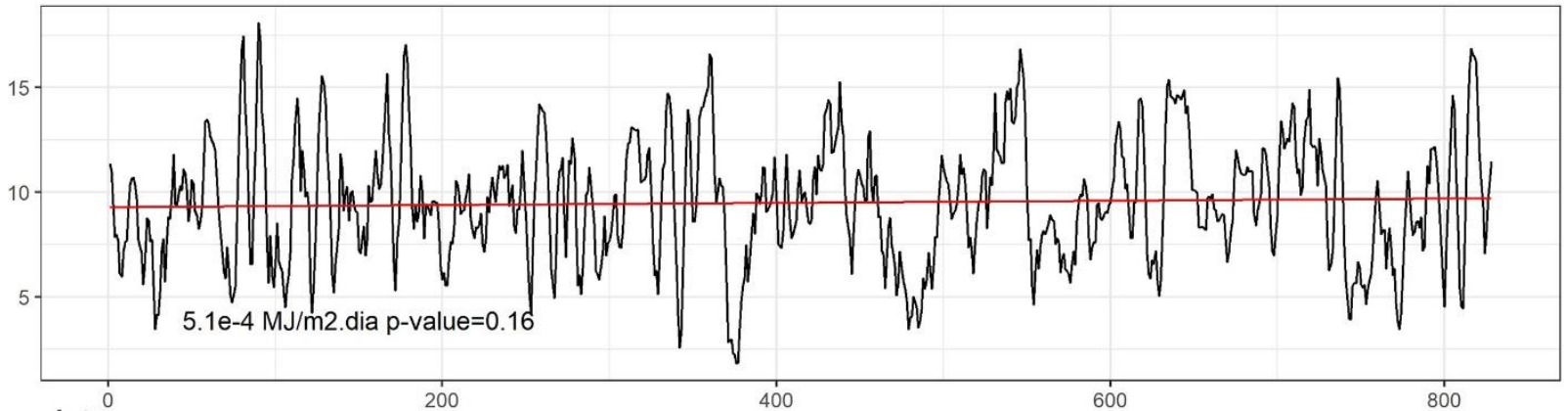

b)

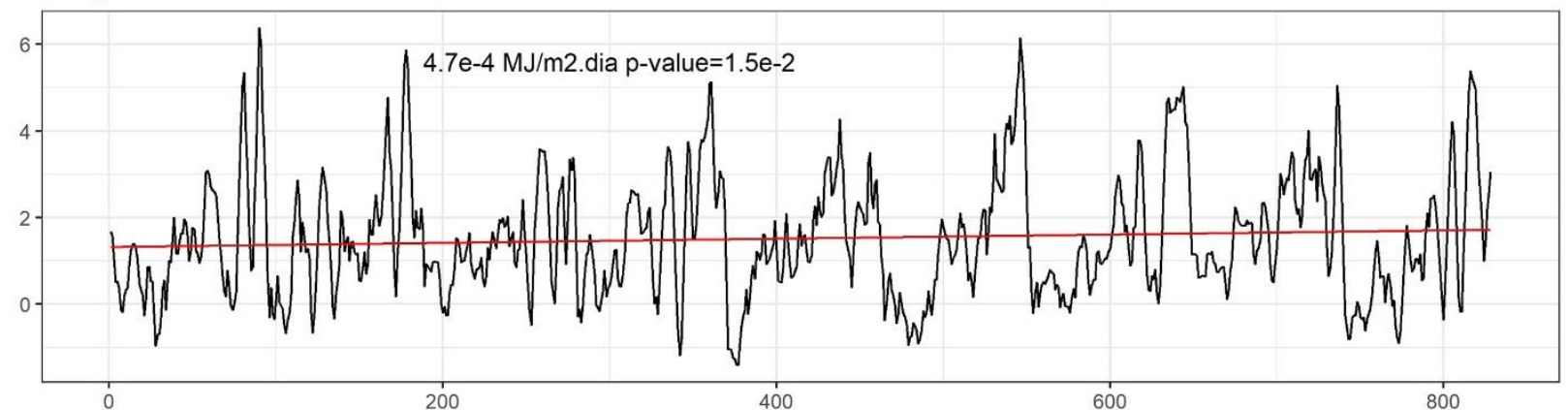

C)

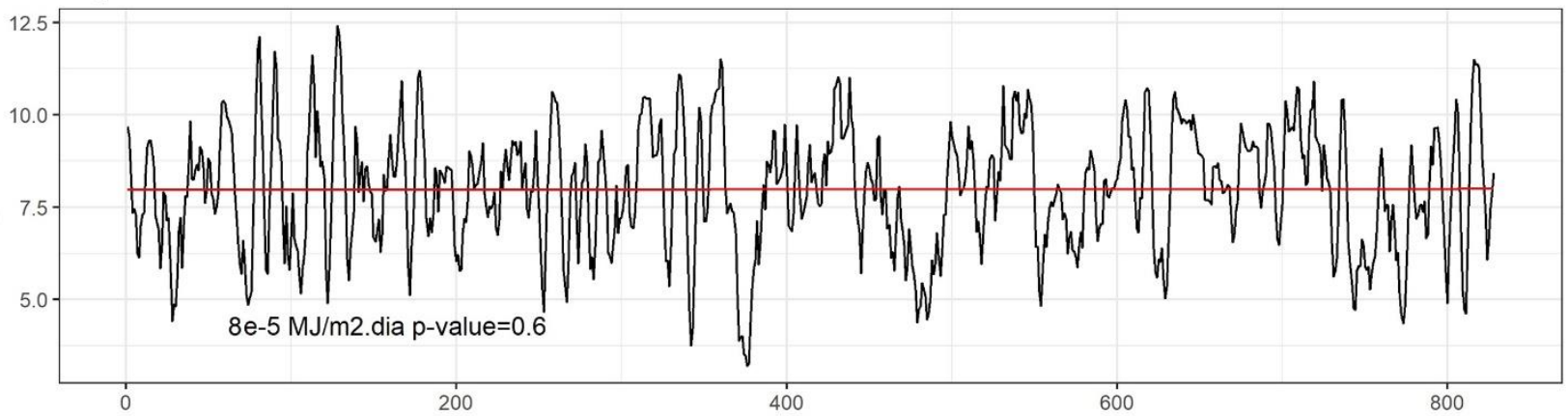

Figura 3. Tendência de (a) Balanço de Ondas Longas, (b) Balanço de Ondas Curtas e (c) Saldo de Radiação, no eixo y, em MJ/m2dia-1 durante os invernos em Cruz Alta no período 2010-2018 (em número de dias contínuos no eixo $\mathrm{x}$ ). 
a)

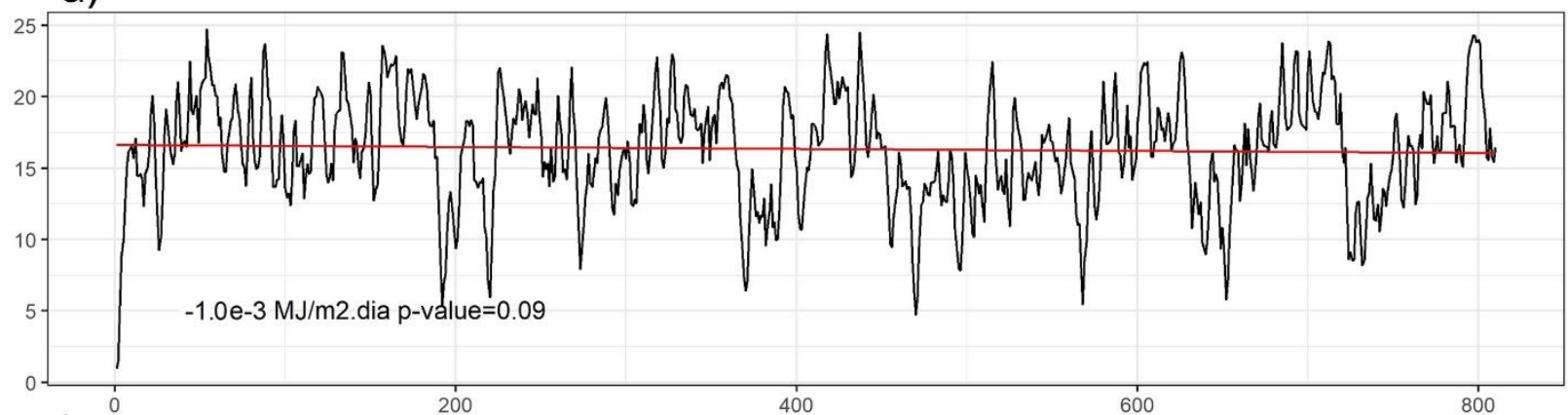

b)

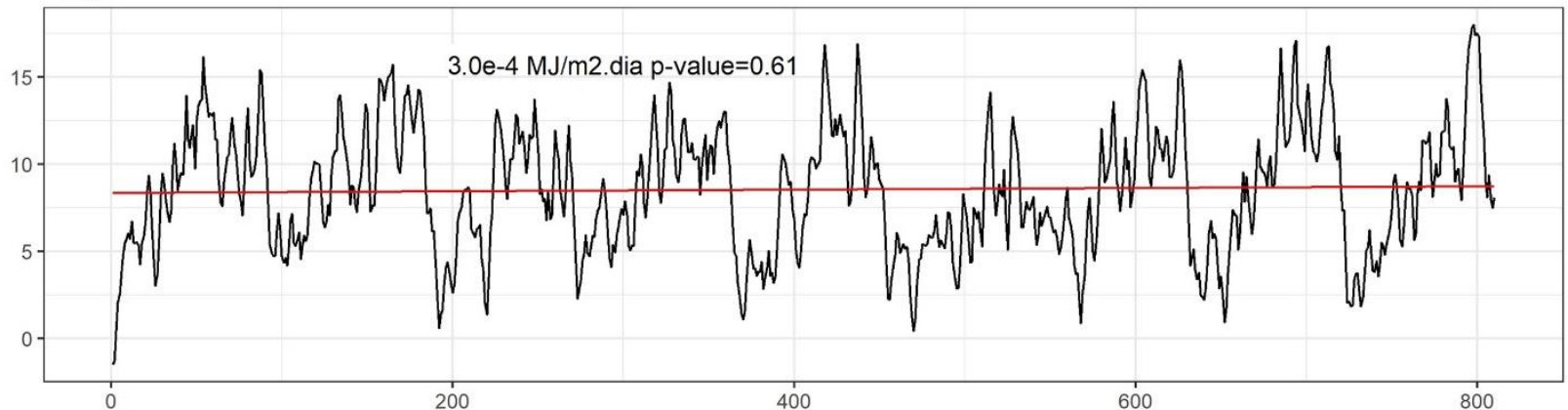

c)

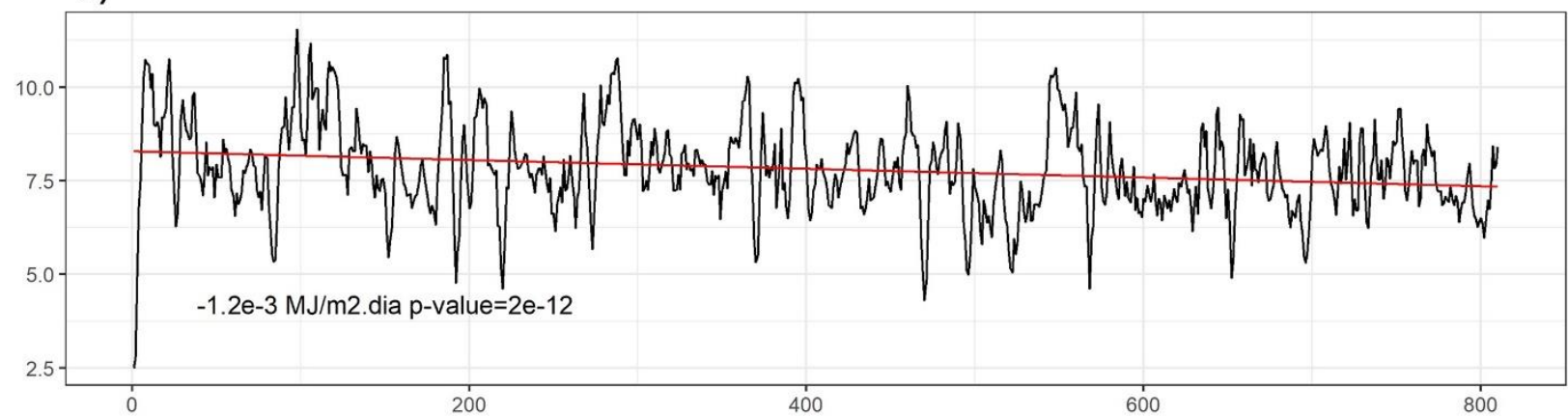

Figura 4. Tendência de (a) Balanço de Ondas Longas, (b) Balanço de Ondas Curtas e (c) Saldo de Radiação, no eixo y, em MJ/m2dia-1 durante as primaveras em Cruz Alta no período 2010-2018 (em número de dias contínuos no eixo $\mathrm{x})$. 
a)

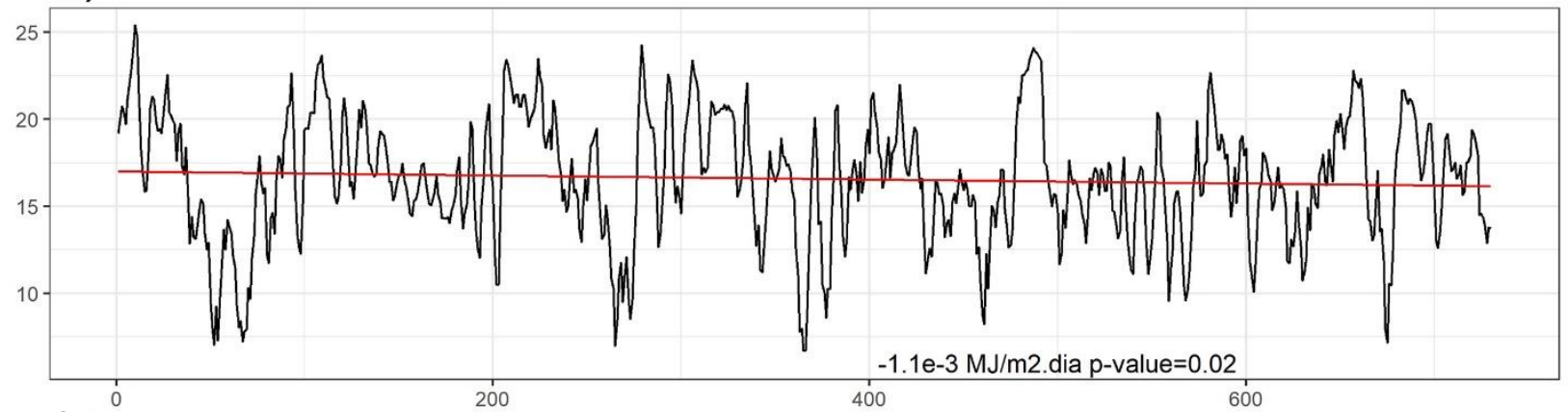

b)

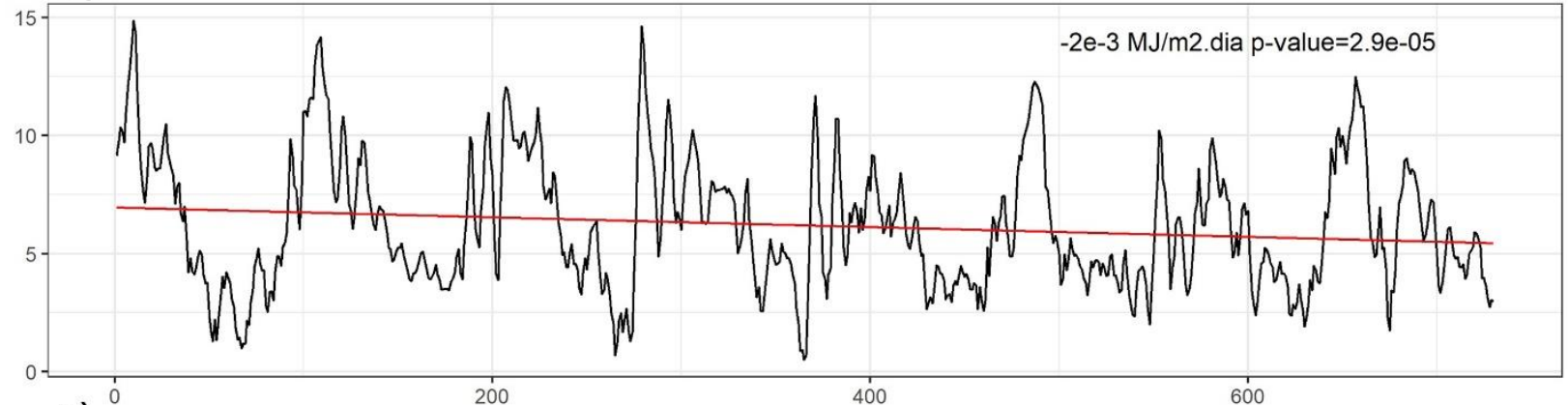

c)

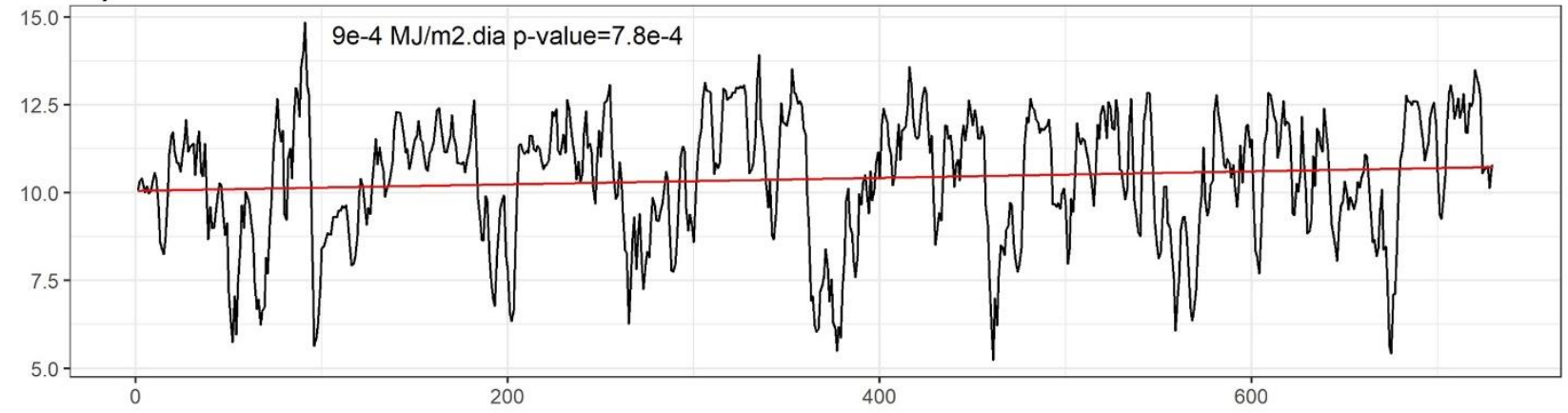

Figura 5. Tendência de (a) Balanço de Ondas Longas, (b) Balanço de Ondas Curtas e (c) Saldo de Radiação, no eixo y, em MJ/m2dia-1 durante os verões em Cruz Alta no período 2010-2018 (em número de dias contínuos no eixo $\mathrm{x})$. 
a)
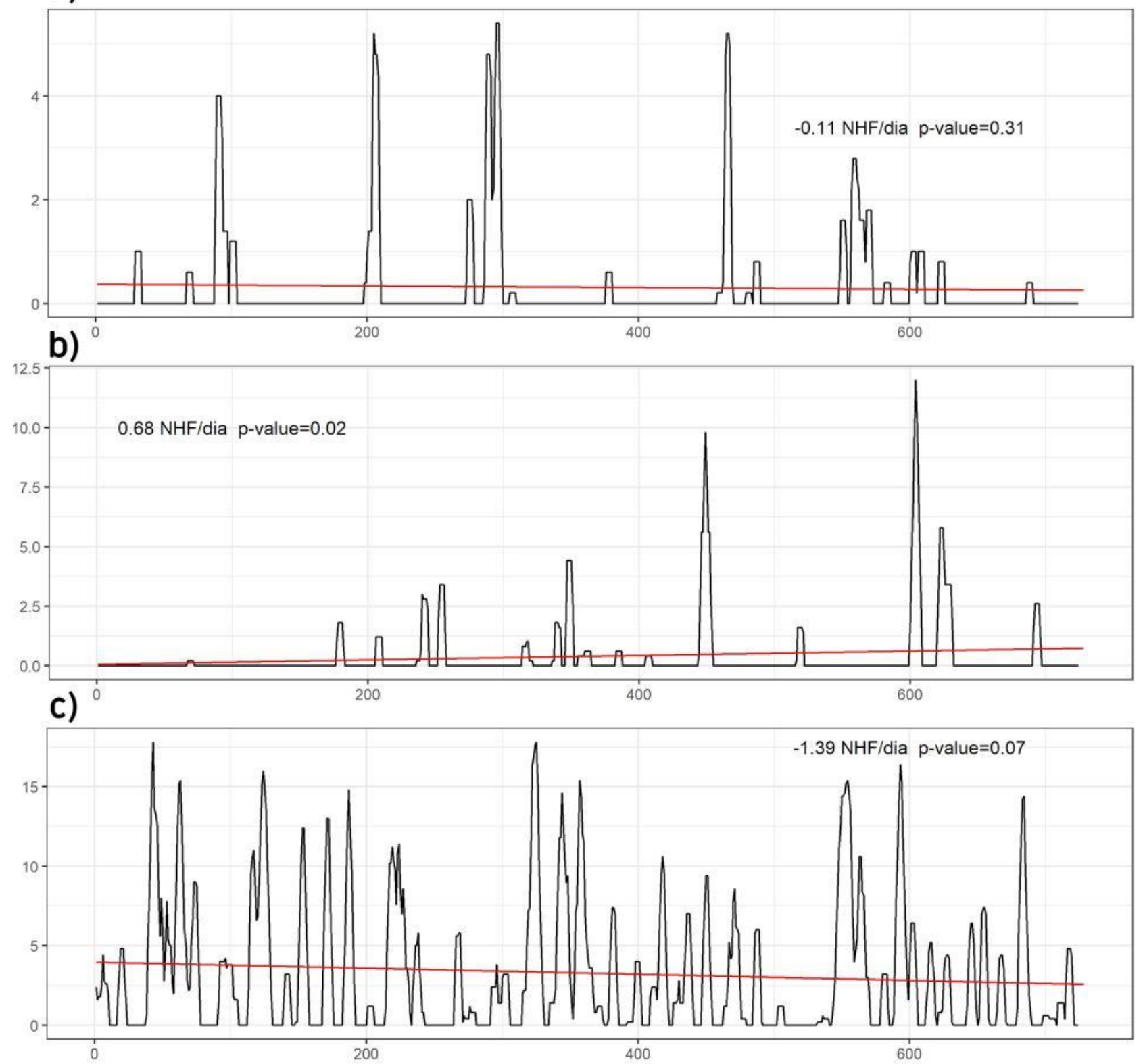

Figura 6. Tendência do Número de Horas de Frio durante a (a) Primavera, (b) Outono e (c) Inverno em Cruz Alta-RS, no eixo y, no período 2010-2018 (em número de dias contínuos no eixo x). 

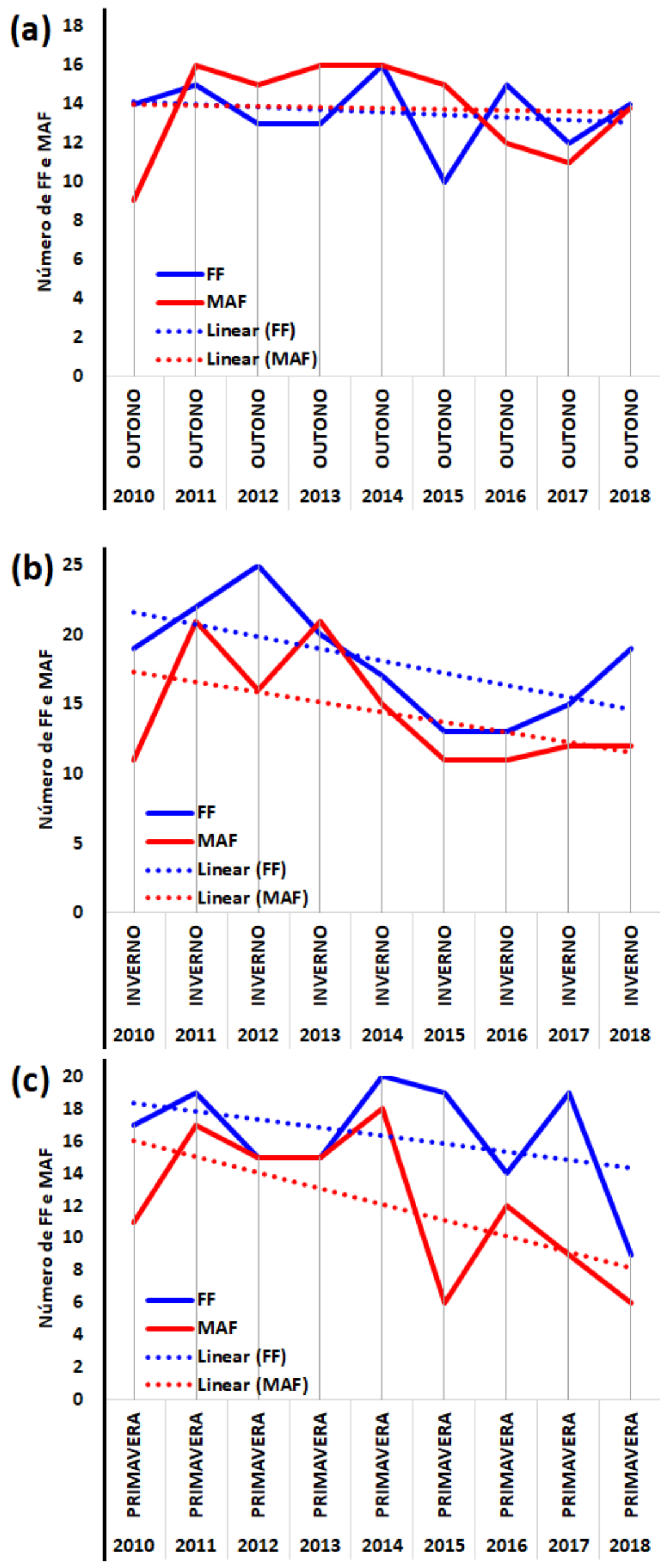
Figura 7. Número e tendências lineares de Frentes Frias e Massas de Ar Frio por Estação do Ano: (a) Outono, (b) Inverno e (c) Primavera. Fontes: CPTEC/INPE (Boletins climanálise) e INMET (Boletins Agrometeorológicos).

\section{Conclusões}

O BOC e o BOL apresentaram tendências positivas no inverno, negativas no outono e verão, e na primavera o BOC mostrou tendência negativa e o BOL positiva. O aumento simultâneo de BOC e BOL no inverno implicou em tendência positiva do saldo de radiação, que teve tendência negativa estatisticamente significativa no outono e na primavera. Apesar da tendência não apresentar significância estatística no inverno, a diminuição do saldo de radiação na principal estação do ano para acúmulo de horas de frio por diferentes culturas, resultou na tendência negativa de NHF mais acentuada nesta estação, com uma diminuição de $-1,4 \mathrm{NHF} / \mathrm{dia} / \mathrm{ano}$, entre 2010 e 2018.

A estação do outono apresentou tendência positiva de $0,68 \mathrm{NHF} /$ dia/ano, com tendência quase linear e sem significância no número de passagens de FF e MAF foi, diferente das estações do inverno e primavera, cuja diminuição significativa da atuação de FF e MAF foi determinante para a redução do NHF.

De modo geral, a tendência observada no inverno e primavera mostra que houve condições propícias ao menor acúmulo de horas de frio nesta estação, o que é desfavorável para diversas culturas que precisam desta situação para sua regulação hormonal e, assim, manter os padrões de produtividade.

\section{Agradecimentos}

$\mathrm{O}$ primeiro autor agradece à Coordenação de Aperfeiçoamento de Pessoal de Nível Superior pela concessão da Bolsa de Mestrado, O terceiro autor agradece à Coordenação de Aperfeiçoamento de Pessoal de Nível Superior pela concessão da Bolsa de Pós-Doutorado Júnior, e o quinto autor agradece a bolsa de iniciação científica concedida pela UFAL/CNPq.

\section{Referências}

Angelini, L. P., Silva, P. C. B. S., Fausto, M. A., Machado, N. G., Biudes, M. S., 2017. Balanço de Energia nas Condições de Mudanças de Uso do Solo na Região Sul do Estado de Mato Grosso. Revista Brasileira de Meteorologia 32, 353-363.
Baldocchi, D., Wong, S., 2008 Accumulated winter chill is decreasing in the fruit growing regions of California. Climatic Change 87, 53-166.

Bianchi, V. J., Arruda, J. J. P., Casagrande, J. G., Herter, F. G., 2000. Estudo da para dormência em pereira por meio do método biológico. Revista Brasileira de Fruticultura 22, 294-296.

Biudes, M. S., Vourlitis, G. L., Machado, N. G., Arruda, P. H. Z., Neves, G. A. R., Lobo, F. A., Neale, C. M. U., Nogueira, J. S., 2015. Patterns of energy Exchange for tropical ecosystems across a climate gradiente in Mato Grosso, Brazil. Agricultural and Forest Meteorology 202, 112-124.

Blad, B. L., Baker, D. G., 1972. Reflected radiation from a soybean crop. Agronomy Journal 64, 277-280.

Botelho, R. V., Ayub, R. A., Muller, M. M. L., 2006. Somatória de horas de frio e de unidades de frio em diferentes regiões do Estado do Paraná. Scientia Agraria 7, 89-96.

Bradley L., Maurer, M., 2002. Deciduous Fruit \& Nuts for the Low Desert. College of AgriAgriculture, The University of Arizona.

Citadin, I., Raseira, M. C. B., Herter, F. G., Silveira, C. A. P., 2002. Avaliação da necessidade de frio em pessegueiro. Revista Brasileira de Fruticultura 24, 703-706.

Cunha, J. E. B. L., Rufino, L. A. A., Bernado, B. S., Chaves, L. B., 2012. Dinâmica da cobertura vegetal para a Bacia de São João do Rio do Peixe, PB, utilizando-se sensoriamento remoto. Revista Brasileira de Engenharia Agrícola e Ambiental 16, 539-548.

Fausto, M. A., Machado, N. G., Nogueira, J. S., Biudes, M. S., 2014 Net radiation estimated by remote sensing in Cerrado areas in the Upper Paraguay River Basin. Journal of Applied Remote Sensing 8, 083541.

Fontana, D. C., Oliveira, D., 1996. Relação entre radiação solar global e insolação no Estado do 
Rio Grande do Sul. Revista Brasileira de Agrometeorologia 4, 87-91.

Geirinhas, J. L., Trigo, R. M., Libonati, R., Coelho, C. A. S., Palmeira, A. C. Climatic and synoptic characterization of heat waves in Brazil. International Journal of Climatology. DOI: 10.1002/joc.5294, 2017.

Hauage, R., Cummins, J.N., 2000. Pome fruit genetic pool for production in warm climates. In: Temperate fruit crops in warm climates. Boston: Kluwer Academic Publishers. Boston, Cap. 10, $267-303$.

Leite, G. B., Bonhomme, M., Rageau, R., Lacointe, A., Sakr, S., Guilliot, A., Maurel, K., Petel, G., Rodrigues, A. C., 2004. Influence of lack of chilling on bud-break patterns and evolution of sugar contents in buds and stem tissues along the one-year-old shoot of the peach trees. Acta Horticulturae, The Hague 662, 61-71.

Luedeling, E., Gebauer, J., Buerkert, A. Climate change effects on winter chill for tree crops with chilling requirements on the Arabian Peninsula. Climatic Change, 2008. doi 10.1007/s10584009-9581-7.

Machiwal, D., Jha, M. K., 2008. Comparative Evaluation of Statistical Tests for Time Series Analysis: Application to Hydrological Time Series. Hydrological Sciences Journal 7, 353366.

Martin, O., 2012. Reliable Fruit Tree Varieties for Santa Cruz County. News \& Notes of the UCSC Farm \& Garden, 132.

Matins, A. L., Cunha, C. R., Pereira, V. M. R., Danelichen, V. H. M., Machado, N. G., 2015. Mudanças em índices biofísicos devido à alteração da cobertura do solo em área nativa de Cerrado em Mato Grosso. Ciência e Natura 37, 152-159.

Milech, C. G., Scariotto, S., Dini, M., Herter, F. G., Raseira, M. C. B, 2008. Models to estimate chilling accumulation under subtropical climatic conditions in Brazil. Revista Brasileira de Climatologia 23, p. 106-115, 2018.

Modarres, R., Silva, V. P. R., 2007. Rainfall trends in arid and semi-arid regions of Iran. Journal of Arid Environments 70, 344-355.
Nachtigall, G. R. (Ed.)., 2004. Maçã: produção. Bento Gonçalves: Embrapa Uva e Vinho; Brasília, DF: Embrapa Informação Tecnológica, $171 \mathrm{p}$.

Nienow, A. A., Floss, L.G., 2002. Florescimento de pessegueiros e nectarineiras no planalto médio do Rio Grande do Sul, influenciada pelas condições meteorológicas. Ciência Rural 32, 931-936.

Onoz, B., Bayazit, M., 2003. The Power of Statistical Tests for Trend Detection. Turkish Journal of Engineering and Environmental Sciences 27, 247-251.

Pedro Júnior, M. J., Ortolani, A. A., Rigitano, O., Alfonsi, R. R., Pinto, H. S., Brunini, O., 1979. Estimativa de horas de frio abaixo de 7 e de $13^{\circ} \mathrm{c}$ para regionalização da fruticultura de clima temperado no Estado de São Paulo. Bragantia $38,123-130$.

Pedro Júnior, M. J., Barbosa, W., Rolim, G. S., Castro, J. L., 2007. Época de florescimento e horas de frio para pessegueiros e nectaríferas. Revista Brasileira de Fruticultura 29, 425-430.

Richard, O. G., 1987. Statistical methods for environmental pollution monitoring. John Wiley \& Sons.

Salmi, T., Määttä, A., Anttila, P., Ruoho-Airola, T., Amnell, T., 2002. Detecting trends of annual values of atmospheric pollutants by the MannKendall test and Sen's slope estimates - the Excel template application MAKESENS. Helsink. Publications on Air Quality No. 31, Report code FMI-AQ-31.

Santos, A. P. P., Aragão, M. R. S., Correia, M. F., Santos, S. R. Q., Silva, F. D. S., Araújo, H. A., 2016. Precipitação na Cidade de Salvador: Variabilidade Temporal e Classificação em Quantis. Revista Brasileira de Meteorologia 31, 454-467.

Sen, P. K., 1968. Estimates of the regression coefficient based on Kendall's tau. Journal of the American Statistical Association 63, 13791389.

SOME'e, B. S., Ezani, A., Tabari, H., 2013. Spatiotemporal trends of aridity index in arid and semi-arid regions of Iran. Theoretical Applied of Climatology 111, 149-160. 
Revista Brasileira de Geografia Física v.13, n.01 (2020) 256-270.

Wilks, D. S., 2011. Statistical methods in the atmospheric sciences. Academic Press, 676p.

Yoshikawa, E. R., Petri, J. L., Sezerino, A. A., 2018. Comparison of chilling units and hours for apple production in Brazil and in Japan. Revista Eletrônica Científica da UERGS 4, 91106. 